
\title{
25 Research Square \\ Sex Differences in Brain Structure Account for the Sexual Distinction on Balanced Time Perspective
}

\section{Tao Chen}

Institute of Psychology Chinese Academy of Sciences

\section{Zhi Li}

Institute of Psychology Chinese Academy of Sciences

Ji-fang Cui

National Institute of Education Sciences

Jia Huang

Institute of Psychology Chinese Academy of Sciences

Muireann Irish

The University of Sydney Brain and Mind Centre

Ya Wang ( $\nabla$ wangyazsu@gmail.com )

Institute of Psychology, Chinese Academy of Sciences https://orcid.org/0000-0001-5764-6740

Raymond Chan

Institute of Psychology Chinese Academy of Sciences

\section{Research Article}

Keywords: Balanced time perspective, executive control, default mode network, grey matter volume, basal ganglia

Posted Date: December 27th, 2021

DOI: https://doi.org/10.21203/rs.3.rs-1174014/v1

License: (c) (i) This work is licensed under a Creative Commons Attribution 4.0 International License.

Read Full License 


\section{Abstract}

Sex differences in behaviour and cognition have been widely observed, however, little is known about such differences in maintaining a balanced time perspective or their potential underlying neural substrates. To answer the above questions, two studies were conducted. In Study 1, time perspective was assessed in 1,913 college students, including 771 males and 1,092 females, and demonstrated that females had a significantly more balanced time perspective than males. In Study 2, 58 males and 47 females underwent assessment of time perspective and structural brain imaging. Voxel-based morphometry analysis and cortical thickness analysis were used to analyse the structural imaging data. Results showed that compared with males, females demonstrated a more balanced time perspective, which primarily related to lower grey matter volume in left precuneus, right cerebellum, right putamen and left supplementary motor area. Analysis of cortical thickness failed to reveal any significant sex differences. Furthermore, the sex difference in grey matter volume of left precuneus, right cerebellum, right putamen and left supplementary motor area could account for the difference in balanced time perspective between males and females. The findings deepen our understanding of sex differences in human cognition and their potential neural signature, and may inform tailored interventions to support a balanced time perspective in daily life.

\section{Introduction}

Mounting evidence points to the importance of studying human subjective temporality and its implications for adaptive and maladaptive thoughts and behaviours in everyday life (for a review, see Stolarski, Zajenkowski, Jankowski, \& Szymaniak, 2020). Time perspective (TP) refers to the "nonconscious process whereby the continual flows of personal and social experiences are assigned to temporal categories, or time frames, that help to give order, coherence, and meaning to those events" (p.1271) (Zimbardo \& Boyd, 1999). According to this original definition, TP is posited to included five dimensions: Future, Past-Negative, Past-Positive, Present-Fatalistic and Present-Hedonistic.

A balanced time perspective is defined as "the mental ability to switch flexibly among TPs depending on task features, situational considerations, and personal resources rather than be biased toward a specific TP that is not adaptive across situations" (Boniwell \& Zimbardo, 2004). Proposed as a central ingredient of positive psychology (Boniwell \& Zimbardo, 2004), the beneficial effects of a balanced time perspective have been observed across a many aspects of subjective well-being (T. Chen et al., 2020; Zhang, Howell, \& Stolarski, 2013), including affective regulation (McKay et al., 2019), romantic relationships (Stolarski, Wojtkowska, \& Kwiecinska, 2016) and mindfulness (Rönnlund et al., 2019; Stolarski, Vowinckel, Jankowski, \& Zajenkowski, 2016). As such, it is important to delineate the mechanisms that support a balanced time perspective as well as potential factors that may constrain or influence its expression.

Sex differences represent a particularly important line of enquiry in this regard, and have long held the interest of scientists and the general public alike (Jazin \& Cahill, 2010). The literature is replete with findings suggesting significant sex differences across multiple domains including human executive 
function (Gur \& Gur, 2017; Gur et al., 2012), social cognition (Christov-Moore et al., 2014; Ingalhalikar et al., 2014; Proverbio, 2021) and personality traits (Costa, Terracciano, \& McCrae, 2001). In general, males are generally overshadowed by females on tests of nonverbal reasoning and emotion identification, however the converse profile is typically observed on tests of motor and spatial cognition (Satterthwaite et al., 2014). At present, sex was generally controlled as a covariate in studies exploring balanced time perspective (Stolarski et al., 2020), which makes the sex difference on balanced time perspective unclear. Little is known about sex differences in balanced time perspective and the available evidence to date is mixed. Two studies have reported that females are more balanced in time perspective than males with a level, however effects have ranged from significant to marginal (T. Chen, Liu, Cui, Chen, \& Wang, 2016; Guo, Chen, \& Feng, 2017). In contrast, a separate study showed females were significantly less balanced in time perspective than males $(N=140, p=0.032)(\mathrm{Wu}, Z$ hou, Zhao, Qiu, \& Guo, 2019). Given these conflicting findings, it is important to explore this question using a large sample.

Determining the neural substrates of maintaining a balanced time perspective remains an active topic of investigation (Guo et al., 2017; Wu et al., 2019). Accumulating evidence suggests a pivotal role for executive functions (e.g., inhibition) in this process (Zajenkowski, Stolarski, Witowska, Maciantowicz, \& Łowicki, 2016) enabling the individual to flexibly switch between different perspectives, and to inhibit potentially maladaptive time perspectives. Thus, it is possible that brain regions supporting executive control (e.g., dorsal lateral prefrontal cortex) (Niendam et al., 2012; Vincent, Kahn, Snyder, Raichle, \& Buckner, 2008), may be implicated in balanced time perspective. In addition, some empirical studies have pointed to the importance of default mode network structures, such as the precuneus, in supporting a balanced time perspective (Guo et al., 2017; Wu et al., 2019). Interestingly, sex differences have been reported in brain regions implicated in cognitive control and regions within the default mode network (Liu, Seidlitz, Blumenthal, Clasen, \& Raznahan, 2020; Luders et al., 2004; Xin, Zhang, Tang, \& Yang, 2019). Whether these differences account for the sex difference in balanced time perspective remains unclear.

In this context, it is important to understand whether the sex difference in balanced time perspective reflects underlying sex differences at the neural level. Mounting evidence converges to suggest considerable sex differences in brain anatomy and function (Gur \& Gur, 2017). Compared with females, males are reported to have larger grey matter volume in subcortical temporal structures (including hippocampus) and motor areas (Lotze et al., 2019). In contrast, males have been shown to display thinner cortical thickness and less gyrification in frontal, parietal regions (Luders et al., 2004; Lv et al., 2010) as well as weaker structural connectivity in the inter-hemispheric connections (Tunç et al., 2016). As such, while there appears to be significant sex differences in terms of underlying brain structure, it remains unclear how such differences relate to the sex difference observed in studies of balanced time perspective.

To address these questions, we designed two studies. Study 1 aimed to confirm that females showed more a balanced time perspective than males with a large sample. As an extension to Study 1, Study 2 explored how potential sex differences in brain anatomy, indexed by grey matter volume and cortex thickness, might account for a more balanced time perspective in females relative to males. 


\section{Study 1}

Study 1 was designed to test the hypothesis that females manifested a more balanced time perspective than males within a large sample.

\section{Method}

\section{Participants}

A total of 1913 college students were recruited from Beijing by advertisement to complete a questionnaire measuring time perspective. The sample included 796 males (mean age $=20.07$ years, $S D=1.16$ ) and 1117 females (mean age $=19.75$ years, SD = 1.11), in which age information of 24 males and 25 females was missing. Participants were required to complete the questionnaire after a brief introduction to the study. Written informed consent was provided by participants before the commencement of the study. This study was approved by the ethics committee of the Institute of Psychology, Chinese Academy of Sciences.

\section{Measures}

The Chinese brief version of the Zimbardo Time Perspective Inventory (ZTPI) was used to measure time perspective (Wang, Chen, Cui, \& Liu, 2015; Zimbardo \& Boyd, 1999). The 20-item Chinese version of ZTPI includes five dimensions: Future, Past-Negative, Past-Positive, Present-Fatalistic and Present-Hedonistic. Participants were required to respond to each item on a 5-point scale ranging from 1 (very untrue) to 5 (very true). The 20 -item Chinese version of ZTPI displays good psychological properties (Wang et al., 2015).

Deviation from the Balanced Time Perspective (DBTP) ${ }^{[1]}$ indicates how far an individual's time perspective profile deviates from the optimal time perspective profile. Derived from the ZPTI, DBTP is proposed as the optimal indicator of a balanced time perspective, with lower scores denoting greater balance (Zhang et al., 2013). DBTP has been widely adopted in the Chinese population (T. Chen et al., 2016; T. Chen et al., 2020; Guo et al., 2017; Wu et al., 2019).

\section{Data analysis}

DBTP was not normally distributed in males (Shapiro-Wilk W $=0.987, p<0.001$ ) or females (Shapiro-Wilk $W=0.980, p<0.001$ ), thus the Mann-Whitney $U$ test was conducted to explore differences in DBTP between males and females.

\section{Results}

As expected, compared with males $(M=2.29, S D=0.83)$, females $(M=2.15, S D=0.75)$ showed more balanced time perspective (Mann-Whitney $\mathrm{U}=400942, p<0.001$, Cohen' $d=0.181$ ) (see Fig. 1).

\section{Footnote:}




$$
{ }_{1} D B T P=\sqrt{(o P N-e P N)^{2}+(o P P-e P P)^{2}+(o P F-e P F)^{2}+(o P H-e P H)^{2}+(o F-e F)^{2}}
$$

oPN (1.95), oPP (4.60), oPF (1.50), oPH (3.90), oF (4.00) were optimal scores of Past-Negative, PastPositive, Present-Fatalistic, Present-Hedonistic, and Future time perspective, respectively. ePN, ePP, ePF, ePH, eF were empirical scores reported by participants (Stolarski, Wiberg, \& Osin, 2015).

\section{Study 2 - Neural Basis Of Sex Differences In Balanced Time Perspective}

Study 2 aimed to examine potential sex differences in grey matter volume and cortical thickness, and to test whether neuroanatomical differences could account for a more balanced time perspective in females relative to males.

\section{Method}

\section{Participants and procedure}

A total of 105 participants, including 58 males (mean age $=19.53$ years, $S D=2.37$ ) and 47 females (mean age $=19.32$ years, $S D=1.46)$, participated in Study 2. No participants reported drug/alcohol abuse/dependence, history of psychiatric/neurological disorders or family history of psychiatric disorders. All participants completed questionnaires first, followed by a structural imaging scan. The study was approved by the ethics committee of the Institute of Psychology, Chinese Academy of Sciences. Written informed consent was provided by participants.

\section{Measures}

The measure of balanced time perspective was the same as Study 1, i.e., DBTP from the ZPTI.

\section{Brain imaging data acquisition}

Structural MRI data were acquired with a Siemens 3T scanner (SIEMENS 3T-Trio A Tim, Erlangen, Germany) using a 32-channel head coil at Hospital 306, Beijing. High-resolution T1-weighted anatomical images were obtained with a Magnetization Prepared Rapid Acquisition Gradient-Echo (MPRAGE) sequence $\left(T R=2300 \mathrm{~ms}, \mathrm{TE}=3.01 \mathrm{~ms}\right.$, flip angle $=9^{\circ}, \mathrm{FOV}=240 \times 256 \mathrm{~mm}$, matrix $=256 \times 256$, voxel size $=1 \times 1 \times 1 \mathrm{~mm})$.

\section{Voxel-based morphometry (VBM) analysis}

Voxel-based morphometry (VBM) analysis was performed with the Statistical Parametric Mapping package (SPM12, https://www.fil.ion.ucl.ac.uk/spm/) and the Computational Anatomy Toolbox 12 (CAT12; http://dbm.neuro.uni-jena.de/cat.html) running in MatLab R2013b (The MathWorks, Inc., Natick, MA, USA). T1-weighted structural images were initially reoriented to the same spatial orientation and the point of the anterior commissure, and followed by pre-processing with standard settings of the CAT12 
toolbox: skull-stripping, bias corrections for magnetic field inhomogeneity, precise segmentation into grey matter, white matter, and cerebrospinal fluid, followed by spatial normalization to the DARTEL template in the Montreal Neurological Institute (MNI) space (voxel size: $1.5 \mathrm{~mm} \times 1.5 \mathrm{~mm} \times 1.5 \mathrm{~mm}$ ). Finally, spatial smoothing was performed with an 8-mm full-width at half maximum isotropic Gaussian kernel. The VBM data was applied with a 0.2 absolute masking threshold. Automated measures of quality control implemented in the CAT12 toolbox and careful visual inspection of the data were applied to ensure data quality.

\section{Surface-based morphometry (SBM) analysis}

Cortical thickness analysis was performed with SPM12 and the CAT12 running in MatLab R2013b (The MathWorks, Inc., Natick, MA, USA). Cortical thickness was estimated using a projection-based methodology, implemented in the CAT12 toolbox, by calculating the distance between the inner and outer cortical surface (Dahnke, Yotter, \& Gaser, 2013). Projection-based thickness can deal well with partial volume information, sulcal blurring, and sulcal asymmetries. Topological correction, spherical mapping, and spherical registration were conducted to derive a cortical thickness index. The merged surface of both hemispheres were then resampled and spatially smoothed with 15-mm full-width at half maximum isotropic Gaussian kernel. To ensure the SBM data quality, automated measures of quality control implemented in the CAT12 toolbox and careful visual inspection of the data were applied.

\section{Data analysis}

Independent $t$ tests were performed with CAT12/SPM12 to examine potential sex differences in grey matter volume and cortical thickness controlling for age. Total intracranial volume (TIV) was additionally controlled for in the VBM analysis. Voxel-level thresholds ( $p<0.001$ without correction) and cluster-level thresholds ( $p<0.05$ with FWE cluster correction for multiple comparisons) were further applied to guard against false positive results. To examine whether sex differences in brain anatomy underlie sex difference in balanced time perspective, we derived the mean values of each significant cluster for brain regions showing a significant sex difference. Those mean grey matter volumes, and cortical thickness values in each significant cluster were separately controlled as covariates in a series of ANCOVAs comparing the sex difference in DBTP.

\section{Results}

There was no sex difference in terms of age $(p=0.587)$. On structural neuroimaging, males were found to have significantly higher TIV relative to females $(p<0.001)$ (see Table 1 ).

DBTP was normally distributed in males (Shapiro-Wilk $W=0.975, p=0.271$ ) and females (Shapiro-Wilk $W=0.952, p=0.053)$. We replicated the finding of significantly lower DBTP in females relative to males $(p=0.013$, Cohen' $d=0.496)$ indicating a more balanced time perspective in females.

VBM analysis showed that, compared with females, males had significantly larger grey matter volume in the right cerebellum (Crus I), left precuneus, left supplementary motor area and right putamen $(p<0.05$, 
FWE cluster-level correction) (see Table 2 and Fig. 2). SBM analysis revealed no sex difference in cortical thickness $(p<0.05$, FWE cluster-level correction).

A series of ANCOVA demonstrated that the significant sex difference in DBTP disappeared when any of the grey matter volumes of the above regions (i.e., left precuneus, right cerebellum (Crus I), right putamen, left supplementary motor) were included as a covariate in the analyses (see Table 3).

\section{General Discussion}

This study sought to explore sex differences in the maintenance of a balanced time perspective and to identify potential neuroanatomical correlates of such differences using structural brain imaging. Across two independent studies, we found evidence of a more balanced time perspective in females compared to males, which related to smaller grey matter volumes in the left precuneus, right cerebellum Crus I, right putamen and left supplementary motor area. Furthermore, controlling for any of the above grey matter volumes was found to ameliorate the significant sex difference in balanced time perspective. Our findings suggest that distinct structural neuroanatomical regions may underlie sex-based differences in the maintenance of a balanced time perspective.

Despite mounting interest in the adaptive utility of maintaining a balanced time perspective, little is known regarding potential sex differences. Notably, our findings of a more balanced time perspective in females relative to their male counterparts are in line with a previous study in an independent sample (T. Chen et al., 2016). The cognitive processes that support this sex-based difference, however, remain unclear. Maintenance of a balanced time perspective is posited to rely on cognitive control processes (e.g., working memory, inhibition), which enable the flexible switching between different temporal orientations (Stolarski, Bitner, \& Zimbardo, 2011; Witowska \& Zajenkowski, 2019; Zajenkowski et al., 2016). Previous studies have reported that females outperform males on tests that index distinct facets of cognitive control, including working memory (Duff \& Hampson, 2001), inhibition (Sjoberg \& Cole, 2018), attention and reasoning ability (Gur et al., 2012). We tentatively suggest that sex differences in cognitive control may drive the differences in maintaining a balanced time perspective, although future studies directly testing this proposal will be required.

The second major aim of our study was to elucidate potential neuroanatomical sex-based differences that could potentially account for the optimal time perspective exhibited by females as compared to males. Our VBM analysis revealed that males had larger TIV, and higher grey matter volumes in left precuneus, right cerebellum (Crus I), right putamen and left supplementary motor area than females when controlling for TIV. In contrast, we did not find sex difference in cortical thickness.

We found that the sex difference in grey matter volume in left precuneus, right cerebellum Crus I, right putamen and left supplementary motor area accounted for the difference in balanced time perspective between males and females. Considering first the precuneus, our results dovetail well with previous studies exploring grey-matter correlates of DBTP in young college students. Two studies have demonstrated that grey matter volume in the precuneus is positively associated with DBTP (Guo et al., 
2017; Wu et al., 2019), whereby individuals with less balanced time perspective had a larger precuneus, although sex-based differences were not explored in these studies. As a key region of the brain's default mode network (DMN) (Raichle et al., 2001; Utevsky, Smith, \& Huettel, 2014), the precuneus supports a number of complex cognitive functions that are essential for self-referential processing and episodic past and future thinking (Cavanna \& Trimble, 2006). The precise role of the precuneus in modulating a balanced time perspective remains unclear. As such, it will be important to delineate how DMN integrity supports the capacity for flexible switching between different time perspectives, and how sex differences impact these switching processes.

Our results further implicated regions traditionally viewed as motor-related regions, including the cerebellum and supplementary motor area, in the origin of sex difference in balance time perspective. At first glance, regions typically associated with motor function might not be expected to support a cognitively demanding process such as a balanced time perspective. However, accumulating evidence from neuroimaging and clinical studies indicates that these regions serve a number of cognitive control functions, such as inhibition (Nachev, Kennard, \& Husain, 2008; Sathyanesan et al., 2019; Strick, Dum, \& Fiez, 2009), most of which have been proposed to underlie balanced time perspective (Zajenkowski et al., 2016). The supplementary motor area is tightly linked with cognitive control (Nachev et al., 2008), with evidence from neuroimaging studies suggesting an association between pre-supplementary motor area and inhibition (Simmonds, Pekar, \& Mostofsky, 2008) and cognitive flexibility (Crone, Wendelken, Donohue, \& Bunge, 2005). Similarly, mounting evidence indicates the cerebellum in cognitive functions including executive control (Bellebaum \& Daum, 2007; E, Chen, Ho, \& Desmond, 2014; Stoodley, 2012), verbal working memory (Ben-Yehudah, Guediche, \& Fiez, 2007; Hayter, Langdon, \& Ramnani, 2007), inhibition (Peterburs \& Desmond, 2016) and cognitive flexibility (De Bartolo et al., 2009; Jung et al., 2019). In addition, cerebellar dysfunction is now understood to impact cognitive and functional decline in clinical populations including neurodegenerative disorders (Y. Chen et al., 2018; Y. Chen, Kumfor, LandinRomero, Irish, \& Piguet, 2019) and schizophrenia (Segarra et al., 2008). Apart from executive control, the cerebellum is increasingly understood to serve an emotion regulation function (Schutter \& Van Honk, 2005). As such, cerebellar involvement may reflect emotion regulation processes which are positively associated with balanced time perspective (Stolarski et al., 2011).

Finally, we found evidence for sex differences in subcortical structures in the origin of sex differences in balanced time perspective. Of note, the putamen emerged as the only subcortical region implicated in sex differences in this study. As a key region of the basal ganglia, the putamen is reliably implicated in the brain's reward pathways (Haber \& Knutson, 2010) as well as motor and cognitive function. Neuroimaging and lesion studies converge to suggest that the putamen contributes to higher cognitive functions implicated in a balanced time perspective including working memory (Voytek \& Knight, 2010), inhibition (Rubia et al., 2006), cognitive flexibility (Monchi, Petrides, Strafella, Worsley, \& Doyon, 2006) and episodic memory (Ystad, Eichele, Lundervold, \& Lundervold, 2010).

Taking our findings together, our results can be interpreted in favour of sex differences in a corticosubcortical-cerebellar network that contributes to sex differences in the maintenance of a balanced time 
perspective (Bostan \& Strick, 2018; Habas et al., 2009). Our findings of an inverse relationship between brain structure and performance warrant further exploration. We tentatively propose that these associations may reflect synaptic or neuronal pruning processes during brain maturation (Kanai \& Rees, 2011). Brain grey matter development has been shown to follow an inverted U-shape with peak total brain volume and grey matter volume in females emerging on average 2 years earlier than males, and decreasing along a steeper slope than males until early adulthood (Lenroot et al., 2007), suggesting earlier neuronal pruning in females. Whether sex-based differences in cortical pruning (e.g., cortical thickness) account for greater efficiencies in balanced time perspective remain unknown, and future studies adopting a longitudinal approach may offer some clarity on this matter.

\section{Conclusions}

In summary, our findings offer preliminary evidence to suggest sex differences in the maintenance of a balanced time perspective can be linked to underlying differences in brain structure. Future research is now required to build on these findings to determine the functional implications of differences in time perspective in terms of subjective wellbeing in males versus females (T. Chen et al., 2020; Zhang et al., 2013). Moreover, given the correlational nature of our study, longitudinal approaches incorporating multiple time points across the lifespan are warranted to determine the precise origin of sex differences in the neural correlates of time perspective. These approaches may lend themselves to novel interventions to improve time perspective in males, which in turn may impact positively on other aspects of subjective wellbeing including mental health.

\section{Declarations}

Author contributions included conception and study design (TC, MI and $\mathrm{YW}$ ), data collection or acquisition (ZL, JH, YW and RCK), statistical analysis (TC, JH, MI), interpretation of results (TC, MI and $\mathrm{YW}$ ), drafting the manuscript work or revising it critically for important intellectual content (TC, JFC, $\mathrm{MI}, \mathrm{YW}$ and RCK) and approval of final version to be published and agreement to be accountable for the integrity and accuracy of all aspects of the work (All authors).

Funding YW is supported by National Science Foundation of China (32071062), MI is supported by an Australian Research Council Future Fellowship (FT160100096), and RC is supported by the Philip KH Wong Foundation.

Data availability The datasets are available from the corresponding author upon request.

Code availability Not applicable.

Ethical approval This study was approved by the ethics committee of the Institute of Psychology, Chinese Academy of Sciences. 
Informed consent Informed consent was collected from all participants, who were paid for their participation.

Consent for publication Participants approved the data to be published.

Conflict of interest All the authors declared that they have no conflict of interest.

\section{References}

1. Bellebaum, C., \& Daum, I. (2007). Cerebellar involvement in executive control. The Cerebellum, 6(3), 184-192. doi:10.1080/14734220601169707

2. Ben-Yehudah, G., Guediche, S., \& Fiez, J. A. (2007). Cerebellar contributions to verbal working memory: beyond cognitive theory. The Cerebellum, 6(3), 193. doi:10.1080/14734220701286195

3. Boniwell, I., \& Zimbardo, P. G. (2004). Balancing Time Perspective in Pursuit of Optimal Functioning Positive Psychology in Practice (pp. 165-178): John Wiley \& Sons, Inc.

4. Bostan, A. C., \& Strick, P. L. (2018). The basal ganglia and the cerebellum: nodes in an integrated network. Nature Reviews Neuroscience, 19(6), 338-350. doi:10.1038/s41583-018-0002-7

5. Cavanna, A. E., \& Trimble, M. R. (2006). The precuneus: a review of its functional anatomy and behavioural correlates. Brain, 129(Pt 3), 564-583. doi:10.1093/brain/awl004

6. Chen, T., Liu, L.-I., Cui, J.-f., Chen, X.-j., \& Wang, Y. (2016). Developmental trajectory of time perspective: From children to older adults. PsyCh Journal, 5(4), 245-255. doi:10.1002/pchj.140

7. Chen, T., Liu, L.I., Cui, J.-f., Qin, X.-j., Shi, H.-S., Irish, M., \& Wang, Y. (2020). Balanced Time Perspective and Life Satisfaction: The Mediating Role of "Temporal Negative Affect". Journal of Happiness Studies. doi:10.1007/s10902-020-00330-9

8. Chen, Y., Kumfor, F., Landin-Romero, R., Irish, M., Hodges, J. R., \& Piguet, O. (2018). Cerebellar atrophy and its contribution to cognition in frontotemporal dementias. Ann Neurol, 84(1), 98-109. doi:10.1002/ana.25271

9. Chen, Y., Kumfor, F., Landin-Romero, R., Irish, M., \& Piguet, O. (2019). The Cerebellum in Frontotemporal Dementia: a Meta-Analysis of Neuroimaging Studies. Neuropsychology Review, 29(4), 450-464. doi:10.1007/s11065-019-09414-7

10. Christov-Moore, L., Simpson, E. A., Coudé, G., Grigaityte, K., lacoboni, M., \& Ferrari, P. F. (2014). Empathy: Gender effects in brain and behavior. Neuroscience \& Biobehavioral Reviews, 46, 604-627. doi:10.1016/j.neubiorev.2014.09.001

11. Costa, P. T., Terracciano, A., \& McCrae, R. R. (2001). Gender differences in personality traits across cultures: robust and surprising findings. Journal of personality and social psychology, 81(2), 322331. doi:10.1037/0022-3514.81.2.322

12. Crone, E. A., Wendelken, C., Donohue, S. E., \& Bunge, S. A. (2005). Neural Evidence for Dissociable Components of Task-switching. Cerebral Cortex, 16(4), 475-486. doi:10.1093/cercor/bhi127 
13. Dahnke, R., Yotter, R. A., \& Gaser, C. (2013). Cortical thickness and central surface estimation. Neurolmage, 65, 336-348. doi:10.1016/j.neuroimage.2012.09.050

14. De Bartolo, P., Mandolesi, L., Federico, F., Foti, F., Cutuli, D., Gelfo, F., \& Petrosini, L. (2009). Cerebellar involvement in cognitive flexibility. Neurobiology of Learning and Memory, 92(3), 310-317. doi:10.1016/j.nlm.2009.03.008

15. Duff, S. J., \& Hampson, E. (2001). A sex difference on a novel spatial working memory task in humans. Brain and cognition, 47(3), 470-493. doi:10.1006/brcg.2001.1326

16. E, K. H., Chen, S. H., Ho, M. H., \& Desmond, J. E. (2014). A meta-analysis of cerebellar contributions to higher cognition from PET and fMRI studies. Human brain mapping, 35(2), 593-615. doi:10.1002/hbm.22194

17. Guo, Y., Chen, Z., \& Feng, T. (2017). Neural substrates underlying balanced time perspective: A combined voxel-based morphometry and resting-state functional connectivity study. Behavioural Brain Research, 332(Supplement C), 237-242. doi:10.1016/j.bbr.2017.06.005

18. Gur, R. C., \& Gur, R. E. (2017). Complementarity of sex differences in brain and behavior: From laterality to multimodal neuroimaging. Journal of Neuroscience Research, 95(1-2), 189-199. doi:10.1002/jnr.23830

19. Gur, R. C., Richard, J., Calkins, M. E., Chiavacci, R., Hansen, J. A., Bilker, W. B., . . Gur, R. E. (2012). Age group and sex differences in performance on a computerized neurocognitive battery in children age 8-21. Neuropsychology, 26(2), 251-265. doi:10.1037/a0026712

20. Habas, C., Kamdar, N., Nguyen, D., Prater, K., Beckmann, C. F., Menon, V., \& Greicius, M. D. (2009). Distinct Cerebellar Contributions to Intrinsic Connectivity Networks. The Journal of Neuroscience, 29(26), 8586-8594. doi:10.1523/jneurosci.1868-09.2009

21. Haber, S. N., \& Knutson, B. (2010). The Reward Circuit: Linking Primate Anatomy and Human Imaging. Neuropsychopharmacology, 35(1), 4-26. doi:10.1038/npp.2009.129

22. Hayter, A. L., Langdon, D. W., \& Ramnani, N. (2007). Cerebellar contributions to working memory. Neurolmage, 36(3), 943-954. doi:10.1016/j.neuroimage.2007.03.011

23. Ingalhalikar, M., Smith, A., Parker, D., Satterthwaite, T. D., Elliott, M. A., Ruparel, K., . . Verma, R. (2014). Sex differences in the structural connectome of the human brain. Proceedings of the National Academy of Sciences, 111(2), 823-828. doi:10.1073/pnas.1316909110

24. Jazin, E., \& Cahill, L. (2010). Sex differences in molecular neuroscience: from fruit flies to humans. Nature Reviews Neuroscience, 11(1), 9-17. doi:10.1038/nrn2754

25. Jung, K.-I., Park, M.-H., Park, B., Kim, S.-Y., Kim, Y. O., Kim, B.-N., . . Song, C.-H. (2019). Cerebellar Gray Matter Volume, Executive Function, and Insomnia: Gender Differences in Adolescents. Scientific Reports, 9(1), 855. doi:10.1038/s41598-018-37154-w

26. Kanai, R., \& Rees, G. (2011). The structural basis of inter-individual differences in human behaviour and cognition. Nature Reviews Neuroscience, 12(4), 231-242. doi:10.1038/nrn3000

27. Lenroot, R. K., Gogtay, N., Greenstein, D. K., Wells, E. M., Wallace, G. L., Clasen, L. S., . . Giedd, J. N. (2007). Sexual dimorphism of brain developmental trajectories during childhood and adolescence. 
Neurolmage, 36(4), 1065-1073. doi:10.1016/j.neuroimage.2007.03.053

28. Liu, S., Seidlitz, J., Blumenthal, J. D., Clasen, L. S., \& Raznahan, A. (2020). Integrative structural, functional, and transcriptomic analyses of sex-biased brain organization in humans. Proceedings of the National Academy of Sciences, 117(31), 18788. doi:10.1073/pnas.1919091117

29. Lotze, M., Domin, M., Gerlach, F. H., Gaser, C., Lueders, E., Schmidt, C. O., \& Neumann, N. (2019). Novel findings from 2,838 Adult Brains on Sex Differences in Gray Matter Brain Volume. Scientific Reports, 9(1), 1671. doi:10.1038/s41598-018-38239-2

30. Luders, E., Narr, K. L., Thompson, P. M., Rex, D. E., Jancke, L., Steinmetz, H., \& Toga, A. W. (2004). Gender differences in cortical complexity. Nature Neuroscience, 7(8), 799-800. doi:10.1038/nn1277

31. Lv, B., Li, J., He, H., Li, M., Zhao, M., Ai, L., ... Wang, Z. (2010). Gender consistency and difference in healthy adults revealed by cortical thickness. Neurolmage, 53(2), 373-382.

doi:10.1016/j.neuroimage.2010.05.020

32. McKay, M. T., Worrell, F. C., Zivkovic, U., Temple, E., Mello, Z. R., Musil, B., . . Perry, J. L. (2019). A balanced time perspective: Is it an exercise in empiricism, and does it relate meaningfully to health and well-being outcomes? International Journal of Psychology, 54(6), 775-785.

doi:10.1002/ijop.12530

33. Monchi, O., Petrides, M., Strafella, A. P., Worsley, K. J., \& Doyon, J. (2006). Functional role of the basal ganglia in the planning and execution of actions. Annals of neurology, 59(2), 257-264. doi:10.1002/ana.20742

34. Nachev, P., Kennard, C., \& Husain, M. (2008). Functional role of the supplementary and presupplementary motor areas. Nature Reviews Neuroscience, 9(11), 856-869. doi:10.1038/nrn2478

35. Niendam, T. A., Laird, A. R., Ray, K. L., Dean, Y. M., Glahn, D. C., \& Carter, C. S. (2012). Meta-analytic evidence for a superordinate cognitive control network subserving diverse executive functions. Cognitive, Affective, \& Behavioral Neuroscience, 12(2), 241-268. doi:10.3758/s13415-011-0083-5

36. Peterburs, J., \& Desmond, J. E. (2016). The role of the human cerebellum in performance monitoring. Current Opinion in Neurobiology, 40, 38-44. doi:10.1016/j.conb.2016.06.011

37. Proverbio, A. M. (2021). Sex differences in the social brain and in social cognition. Journal of Neuroscience Research, n/a(n/a). doi:10.1002/jnr.24787

38. Raichle, M. E., MacLeod, A. M., Snyder, A. Z., Powers, W. J., Gusnard, D. A., \& Shulman, G. L. (2001). A default mode of brain function. Proceedings of the National Academy of Sciences, 98(2), 676. doi:10.1073/pnas.98.2.676

39. Rönnlund, M., Koudriavtseva, A., Germundsjö, L., Eriksson, T., Åström, E., \& Carelli, M. G. (2019). Mindfulness Promotes a More Balanced Time Perspective : Correlational and Intervention-Based Evidence. Mindfulness, 10(8), 1579-1591. doi:10.1007/s12671-019-01113-x

40. Rubia, K., Smith, A. B., Woolley, J., Nosarti, C., Heyman, I., Taylor, E., \& Brammer, M. (2006). Progressive increase of frontostriatal brain activation from childhood to adulthood during eventrelated tasks of cognitive control. Human brain mapping, 27(12), 973-993. doi:10.1002/hbm.20237 
41. Sathyanesan, A., Zhou, J., Scafidi, J., Heck, D. H., Sillitoe, R. V., \& Gallo, V. (2019). Emerging connections between cerebellar development, behaviour and complex brain disorders. Nature Reviews Neuroscience, 20(5), 298-313. doi:10.1038/s41583-019-0152-2

42. Satterthwaite, T. D., Wolf, D. H., Roalf, D. R., Ruparel, K., Erus, G., Vandekar, S., . . Gur, R. C. (2014). Linked Sex Differences in Cognition and Functional Connectivity in Youth. Cerebral Cortex, 25(9), 2383-2394. doi:10.1093/cercor/bhu036

43. Schutter, D. J. L. G., \& Van Honk, J. (2005). The cerebellum on the rise in human emotion. The Cerebellum, 4(4), 290-294. doi:10.1080/14734220500348584

44. Segarra, N., Bernardo, M., Valdes, M., Caldu, X., Falcón, C., Rami, L., . . Junque, C. (2008). Cerebellar deficits in schizophrenia are associated with executive dysfunction. Neuroreport, 19(15), 1513-1517. doi:10.1097/WNR.0b013e3283108bd8

45. Simmonds, D. J., Pekar, J. J., \& Mostofsky, S. H. (2008). Meta-analysis of Go/No-go tasks demonstrating that $\mathrm{fMRI}$ activation associated with response inhibition is task-dependent. Neuropsychologia, 46(1), 224-232. doi:10.1016/j.neuropsychologia.2007.07.015

46. Sjoberg, E. A., \& Cole, G. G. (2018). Sex Differences on the Go/No-Go Test of Inhibition. Archives of Sexual Behavior, 47(2), 537-542. doi:10.1007/s10508-017-1010-9

47. Stolarski, M., Bitner, J., \& Zimbardo, P. G. (2011). Time perspective, emotional intelligence and discounting of delayed awards. Time \& Society, 20(3), 346-363.

48. Stolarski, M., Vowinckel, J., Jankowski, K. S., \& Zajenkowski, M. (2016). Mind the balance, be contented: Balanced time perspective mediates the relationship between mindfulness and life satisfaction. Personality and Individual Differences, 93(Supplement C), 27-31. doi:10.1016/j.paid.2015.09.039

49. Stolarski, M., Wiberg, B., \& Osin, E. (2015). Assessing Temporal Harmony: The Issue of a Balanced Time Perspective. In M. Stolarski, N. Fieulaine, \& W. van Beek (Eds.), Time Perspective Theory; Review, Research and Application: Essays in Honor of Philip G. Zimbardo (pp. 57-71). Cham: Springer International Publishing.

50. Stolarski, M., Wojtkowska, K., \& Kwiecinska, M. (2016). Time for love: Partners' time perspectives predict relationship satisfaction in romantic heterosexual couples. Time \& Society, 25(3), 552-574.

51. Stolarski, M., Zajenkowski, M., Jankowski, K. S., \& Szymaniak, K. (2020). Deviation from the balanced time perspective: A systematic review of empirical relationships with psychological variables. Personality and Individual Differences, 156, 109772. doi:10.1016/j.paid.2019.109772

52. Stoodley, C. J. (2012). The Cerebellum and Cognition: Evidence from Functional Imaging Studies. The Cerebellum, 11(2), 352-365. doi:10.1007/s12311-011-0260-7

53. Strick, P. L., Dum, R. P., \& Fiez, J. A. (2009). Cerebellum and Nonmotor Function. Annual Review of Neuroscience, 32(1), 413-434. doi:10.1146/annurev.neuro.31.060407.125606

54. Tunç, B., Solmaz, B., Parker, D., Satterthwaite, T. D., Elliott, M. A., Calkins, M. E., ... Verma, R. (2016). Establishing a link between sex-related differences in the structural connectome and behaviour. 
Philosophical Transactions of the Royal Society B: Biological Sciences, 371(1688), 20150111. doi:doi:10.1098/rstb.2015.0111

55. Utevsky, A. V., Smith, D. V., \& Huettel, S. A. (2014). Precuneus Is a Functional Core of the Default-Mode Network. The Journal of Neuroscience, 34(3), 932. doi:10.1523/JNEUROSCI.4227-13.2014

56. Vincent, J. L., Kahn, I., Snyder, A. Z., Raichle, M. E., \& Buckner, R. L. (2008). Evidence for a Frontoparietal Control System Revealed by Intrinsic Functional Connectivity. Journal of Neurophysiology, 100(6), 3328-3342. doi:10.1152/jn.90355.2008

57. Voytek, B., \& Knight, R. T. (2010). Prefrontal cortex and basal ganglia contributions to visual working memory. Proceedings of the National Academy of Sciences, 107(42), 18167. doi:10.1073/pnas.1007277107

58. Wang, Y., Chen, X.-j., Cui, J.-f., \& Liu, L.-I. (2015). Testing the Zimbardo Time Perspective Inventory in the Chinese context. Psych J, 4(3), 166-175. doi:10.1002/pchj.103

59. Witowska, J., \& Zajenkowski, M. (2019). Cognitive consequences of timeframe bias. On the link between working memory, cognitive switching, and time perspective. Current Psychology, 1-14.

60. Wu, H., Zhou, R., Zhao, L., Qiu, J., \& Guo, C. (2019). Neural bases underlying the association between balanced time perspective and trait anxiety. Behavioural Brain Research, 359, 206-214. doi:10.1016/j.bbr.2018.10.039

61. Xin, J., Zhang, Y., Tang, Y., \& Yang, Y. (2019). Brain Differences Between Men and Women: Evidence From Deep Learning. Frontiers in Neuroscience, 13(185). doi:10.3389/fnins.2019.00185

62. Ystad, M., Eichele, T., Lundervold, A. J., \& Lundervold, A. (2010). Subcortical functional connectivity and verbal episodic memory in healthy elderly-A resting state fMRI study. Neurolmage, 52(1), 379388. doi:10.1016/j.neuroimage.2010.03.062

63. Zajenkowski, M., Stolarski, M., Witowska, J., Maciantowicz, O., \& Łowicki, P. (2016). Fluid Intelligence as a Mediator of the Relationship between Executive Control and Balanced Time Perspective. Frontiers in Psychology, 7(1844). doi:10.3389/fpsyg.2016.01844

64. Zhang, J. W., Howell, R. T., \& Stolarski, M. (2013). Comparing Three Methods to Measure a Balanced Time Perspective: The Relationship Between a Balanced Time Perspective and Subjective Well-Being. Journal of Happiness Studies, 14(1), 169-184. doi:10.1007/s10902-012-9322-x

65. Zimbardo, P. G., \& Boyd, J. N. (1999). Putting time in perspective: A valid, reliable individualdifferences metric. Journal of Personality and Social Psychology, 77(6), 1271-1288. doi:10.1037/0022-3514.77.6.1271

\section{Tables}

Table 1. DBTP, age and TIV difference between males and females 


\begin{tabular}{llllc} 
& Males $(N=58)$ & Females $(N=47)$ & & \\
& Mean $(S D)$ & Mean $(S D)$ & $t$ & $p$ \\
\hline Age (years) & $19.53(2.37)$ & $19.32(1.46)$ & 0.55 & 0.587 \\
\hline DBTP & $2.31(0.70)$ & $1.93(0.82)$ & 2.53 & 0.013 \\
\hline TIV $\left(\mathrm{mm}^{3}\right)$ & $1556.82(111.13)$ & $1407.46(93.94)$ & 7.33 & $<0.001$
\end{tabular}

Note: DBTP = Deviation from the Balanced Time Perspective; TIV = Total intracranial volume .

Table 2. Regions with larger grey matter volume for males relative to females

\begin{tabular}{llllll}
$\begin{array}{l}\text { Representative } \\
\text { region of the } \\
\text { cluster }\end{array}$ & $\begin{array}{l}\text { Co- } \\
\text { ordinates } \\
\text { of peak } \\
\text { voxel }\end{array}$ & $\begin{array}{l}\text { Cluster } \\
\text { Size } \\
\text { (voxels) }\end{array}$ & \multicolumn{1}{c}{ Anatomical region } & $\begin{array}{c}t \\
\text { value }\end{array}$ \\
\hline Left precuneus & $-2 ; \quad-47 ;$ & 2454 & $\begin{array}{l}\text { Left Precuneus (39\%), Left Posterior cingulate } \\
\text { gyrus (16\%), Right Posterior cingulate gyrus } \\
(14 \%), \text { Right Precuneus (13\%) }\end{array}$ & 5.83 \\
\hline $\begin{array}{l}\text { Right crus I of } \\
\text { cerebellum }\end{array}$ & $\begin{array}{c}38 ; \\
-21\end{array}$ & $-80 ;$ & 3966 & $\begin{array}{l}\text { Right Crus I of cerebellar hemisphere (24\%), Right } \\
\text { Fusiform gyrus (18\%), Right Lobule VI of } \\
\text { cerebellar hemisphere (12\%) }\end{array}$ & 6.58 \\
\hline Right putamen & $27 ;-2 ; 5$ & 807 & $\begin{array}{l}\text { Right Lenticular nucleus-Putamen (69\%), Right } \\
\text { Lenticular nucleus-Pallidum (27\%) }\end{array}$ & 4.21 \\
\hline $\begin{array}{l}\text { Left } \\
\text { supplementary } \\
\text { motor area }\end{array}$ & $0 ;-9 ; 72$ & 1076 & $\begin{array}{l}\text { Left Supplementary motor area (39\%), Left } \\
\text { Paracentral lobule (34\%), Right Supplementary } \\
\text { motor area (22\%) }\end{array}$ & 5.55 \\
\hline
\end{tabular}

Table 3. Sex differences in DBTP after controlling for grey matter volume of regions with sex difference

\begin{tabular}{|lllll|}
\hline Model & Covariate & $\boldsymbol{F}$ & $\boldsymbol{P}$ & $\eta_{\mathbf{p}}{ }^{2}$ \\
\hline 1 & Null & 6.39 & 0.013 & 0.058 \\
2 & Left Precuneus & 0.86 & 0.356 & 0.008 \\
\hline 3 & Right Cerebellum & 0.77 & 0.382 & 0.008 \\
\hline 4 & Right Putamen & 2.90 & 0.092 & 0.028 \\
\hline 5 & Left SMA & 0.10 & 0.758 & 0.001 \\
\hline
\end{tabular}

Note $\triangle \eta_{\mathrm{p}}{ }^{2}=$ partial Eta-squared; SMA = supplementary motor area. 
Figures

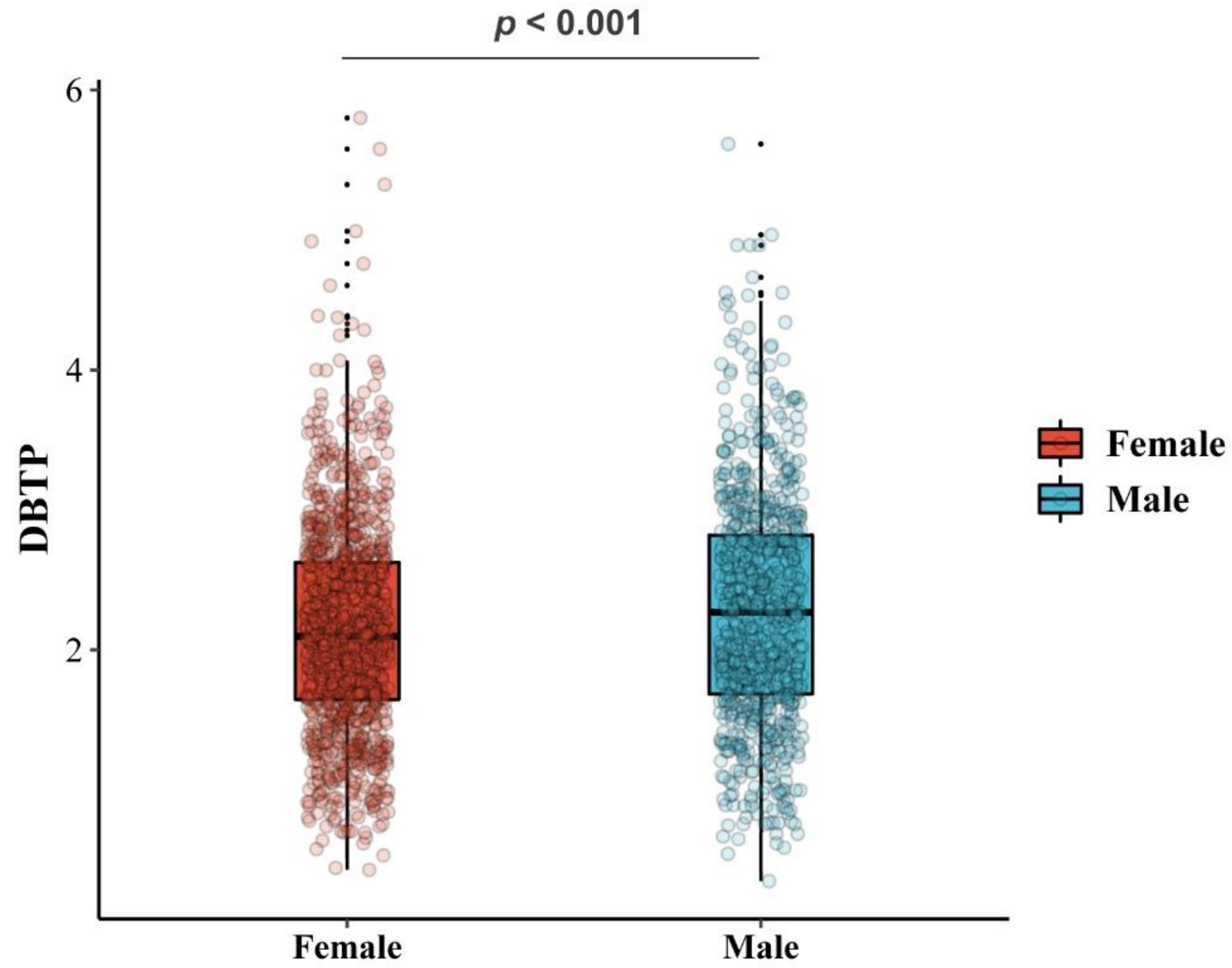

Figure 1

Sex differences in time perspective as measured by DBTP. DBTP $=$ Deviation from the Balanced Time Perspective. 

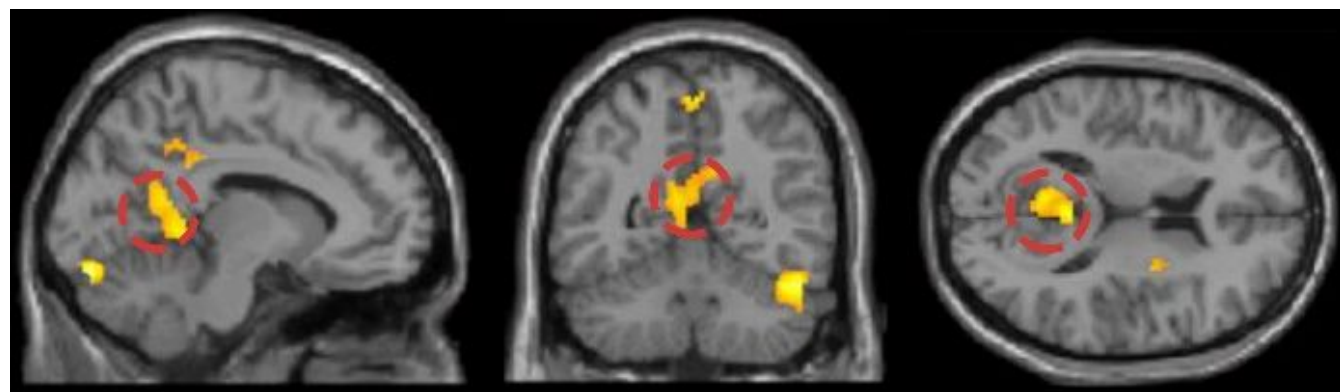

$x=-9.3$

$$
y=-53.6
$$

$$
z=13.3
$$

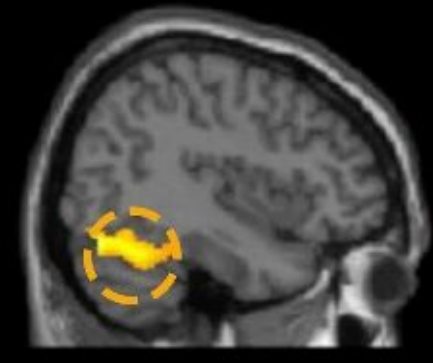

$$
x=41.9
$$

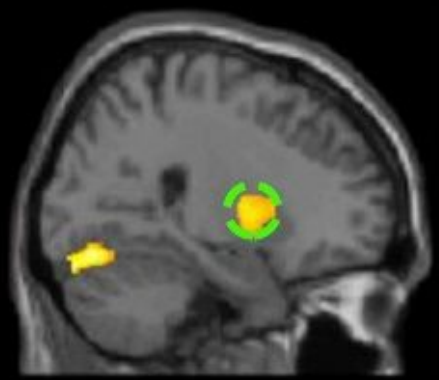

$$
x=24.6
$$

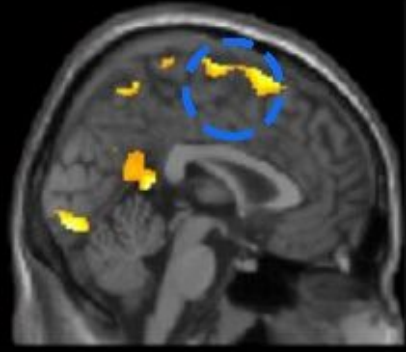

$$
x=1.2
$$

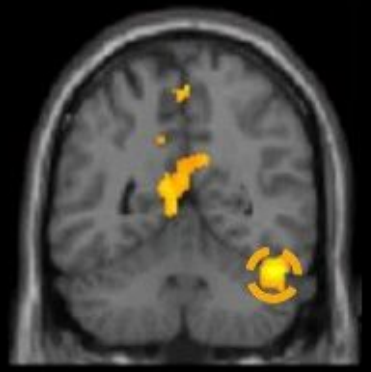

$$
y=-52.3
$$

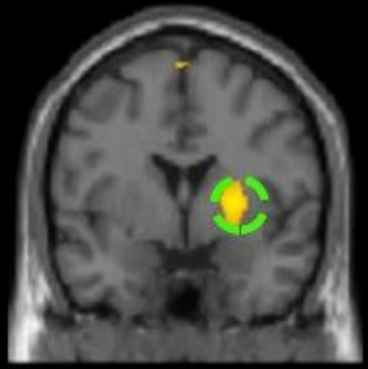

$$
y=0.4
$$

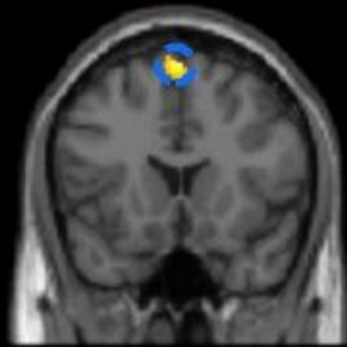

$$
y=17.6
$$

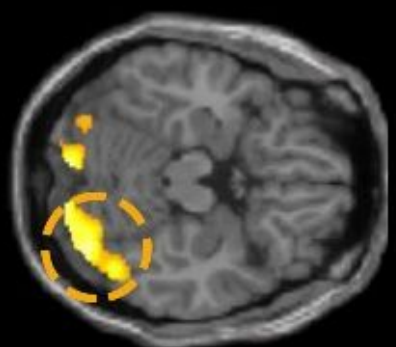

$z=18.2$

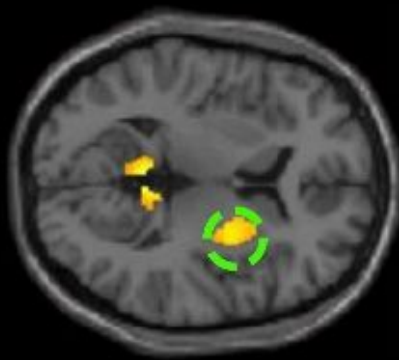

$$
z=6.34
$$

$t$ - value

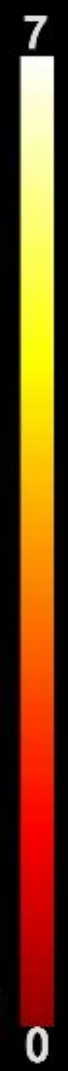

Figure 2

Regions with larger grey matter volume in males relative to females. SMA = supplementary motor area. 